\title{
Phenotypic Multiorgan Involvement of Subclinical Disease as Quantified by Magnetic Resonance Imaging in Subjects With Prediabetes, Diabetes, and Normal Glucose Tolerance
}

\author{
Corinna Storz, MD, * Susanne Rospleszcz, MSc, $†$ Roberto Lorbeer, PhD, $\neq \oint$ Holger Hetterich, MD, $\neq \S$ \\ Sigrid D. Auweter, PhD, $\neq$ Wieland Sommer, MD, $\neq$ Jürgen Machann, PhD, *// $\# \#$ Sergios Gatidis, MD, * \\ Wolfgang Rathmann, MD, ** Margit Heier, MD, † Birgit Linkohr, PhD, † Christa Meisinger, MD, MPH, †† \\ Maximilian Reiser, MD, $\neq$ Udo Hoffmann, MD, MPH, $+\neq$ Annette Peters, PhD, $† \$ \xi \xi$ \\ Christopher L. Schlett, MD, MPH,//// and Fabian Bamberg, MD, MPH* $\$ S$
}

\begin{abstract}
Introduction: Detailed mechanisms in the pathophysiology of diabetes disease are poorly understood, but structural alterations in various organ systems incur an elevated risk for cardiovascular events and adverse outcome. The aim of this study was to compare multiorgan subclinical disease phenotypes by magnetic resonance (MR) imaging to study differences between subjects with prediabetes, diabetes, and normal controls.

Materials and Methods: Subjects without prior cardiovascular disease were enrolled in a prospective case-control study and underwent multiorgan MR for the assessment of metabolic and arteriosclerotic alterations, including age-related white matter changes, hepatic proton density fat fraction, visceral adipose tissue volume, left ventricular remodeling index, carotid plaque, and late gadolinium enhancement. Magnetic resonance features were summarized in a phenotypicbased score (range, 0-6). Univariate, multivariate correlation, and unsupervised clustering were performed.

Results: Among 243 subjects with complete multiorgan MR data sets included in the analysis (55.6 \pm 8.9 years, $62 \%$ males), 48 were classified as subjects with prediabetes and 38 as subjects with diabetes. The MR phenotypic score was significantly higher in subjects with prediabetes and diabetes as compared with controls
\end{abstract}

Received for publication November 7, 2017; and accepted for publication, after revision, December 30, 2017

From the *Department of Diagnostic and Interventional Radiology University of Tuebingen, Tuebingen; †Institute of Epidemiology II, Helmholtz Zentrum Muenchen, German Research Center for Environmental Health, Neuherberg; †Department of Radiology, Ludwig-Maximilians-University-Hospital; §German Center for Cardiovascular Disease Research (DZHK e.V.), Munich; |Institute for Diabetes Research and Metabolic Diseases of the Helmholtz Centre Munich at the University of Tuebingen; $\uparrow$ German Centre for Diabetes Research (DZD); \#Section on Experimental Radiology, Department of Diagnostic and Interventional Radiology, University Hospital Tuebingen, Tuebingen; **Department of Biometry and Epidemiology, German Diabetes Center, Duesseldorf; ††KORA Myocardial Infarction Registry, Central Hospital of Augsburg, Augsburg, Germany; + Cardiac MR PET CT Program, Massachusetts General Hospital, Harvard Medical School, Boston, MA; §§Institute for Cardiovascular Prevention, Ludwig-Maximilians-University-Hospital, Munich; and ||||Department of Diagnostic and Interventional Radiology, University Hospital Heidelberg, Heidelberg, Germany.

Conflicts of interest and sources of funding: This study was funded by the German Research Foundation (DFG, Bonn, Germany), the German Centre for Cardiovascular Disease Research (DZHK, Berlin, Germany), and the German Centre for Diabetes Research (DZD e.V., Neuherberg, Germany).

The KORA study was initiated and financed by the Helmholtz Zentrum München-German Research Center for Environmental Health, which is funded by the German Federal Ministry of Education and Research (BMBF) and by the State of Bavaria.

The authors report no conflicts of interest.

Supplemental digital contents are available for this article. Direct URL citations appear in the printed text and are provided in the HTML and PDF versions of this article on the journal's Web site (www.investigativeradiology.com).

Correspondence to: Fabian Bamberg, MD, MPH, Department of Diagnostic and Interventional Radiology, University of Tuebingen, Hoppe-Seyler-Straße 3, 72076 Tuebingen, Germany. E-mail: Fabian.Bamberg@uni-tuebingen.de.

Copyright (C) 2018 Wolters Kluwer Health, Inc. All rights reserved.

ISSN: $0020-9996 / 18 / 5306-0357$

DOI: $10.1097 /$ RLI.0000000000000451 (mean score, $3.00 \pm 1.04$ and $2.69 \pm 0.98$ vs $1.22 \pm 0.98, P<0.001$ respectively), also after adjustment for potential confounders. We identified 2 clusters of MR phenotype patterns associated with glycemic status $(P<0.001)$, independent of the MR score (cluster II-metabolic specific: odds ratio, $2.49 ; 95 \%$ CI, $1.00-6.17 ; P=0.049$ ). Discussion: Subjects with prediabetes and diabetes have a significantly higher phenotypic-based score with a distinctive multiorgan phenotypic pattern, which may enable improved disease characterization.

Key Words: epidemiology, prediabetes and diabetes, magnetic resonance imaging, risk assessment

(Invest Radiol 2018;53: 357-364)

P revalence of diabetes is steadily increasing throughout developed and developing countries worldwide, representing one of the most common noncommunicable diseases globally with prevalence rates of $7.9 \%$ and $9.0 \%$ in women and men, respectively. ${ }^{1,2}$ As a precursor stage of diabetes, prediabetes affects a substantial proportion of individuals and is defined as an impaired glucose metabolism not satisfying diabetes criteria but also incurring an elevated risk for cardiovascular events and adverse outcome. ${ }^{3}$

Detailed mechanisms in the pathophysiology of prediabetes and diabetes are poorly understood, but structural alterations in various organs are related to the development of impaired glucose metabolism, which in itself is a major risk factor. ${ }^{4,5}$ In addition, previous studies found that nonalcoholic fatty liver disease (NAFLD) may be associated with low-grade chronic inflammatory state, affecting adipose tissue and resulting in abnormal glucose metabolism, increased oxidative stress, dyslipidemia, and endothelial dysfunction with progression of atherosclerosis. ${ }^{6,7}$ Visceral adipose tissue (VAT), in turn, seems to be associated with an increased risk for hypertension, dyslipidemia, and prediabetes. ${ }^{8}$ Furthermore, multiple prior studies demonstrated a correlation between metabolic risk factors and VAT as well as hepatic steatosis in prediabetes and diabetes. $^{9-12}$ In addition to that, white matter changes were previously shown to be associated with cardiovascular risk, cognitive decline, and impaired glucose metabolism and can be detected in patients with metabolic diseases. ${ }^{13-15}$ Thus, the variety of metabolic and organ changes clarify the complex interrelationship of metabolic processes and the development of adverse outcome.

Magnetic resonance (MR) imaging can be used to derive strong prognostic multiorgan phenotypic parameters for the occurrence of metabolic alterations and cardiovascular events in patients with prediabetes and diabetes. ${ }^{9,16,17}$ Relevant parameters include quantification of hepatic fat content by proton density fat fraction (PDFF), VAT, the cerebral age-related white matter changes (ARWMC) score, the left ventricular remodeling index (LVRI), carotid atherosclerotic plaque, as well as post-ischemic changes to the myocardium as evident by late gadolinium enhancement (LGE). ${ }^{15,18-21}$ The noninvasive detection of these MR 
imaging-based parameter may, on one hand, enable a better understanding of the pathophysiology in patients with metabolic diseases, especially in precursor states of diabetes disease, and, on the other hand, allow for an early detection of organic alterations. Summarizing, comprehensive MR imaging provides a detailed assessment of multiorgan alterations in subclinical disease state, which were separately shown to be associated with higher risk profile for adverse cardiovascular outcome. ${ }^{22,23}$ Accordingly, the purpose of our study was to compare multiorgan subclinical disease phenotypes as determined by MR imaging between subjects with prediabetes, diabetes, and controls with normal glucose tolerance and to identify diabetic specific MR pattern differing from subjects with normal glucose tolerance. Our hypothesis was that there is a distinguishable multiorgan phenotypic MR pattern in patients with impaired glucose metabolism compared with subjects with normal glucose tolerance.

\section{MATERIALS AND METHODS}

\section{Study Design}

The study was approved by the institutional review board and all participants provided written informed consent.

The study was designed as a prospective case control study nested in a cohort from the Cooperative Health Research in the Region of Augsburg (KORA). As described elsewhere, subjects were recruited from the FF4 follow-up of the KORA study, representing a large sample from the general population in the region of Augsburg, Germany. ${ }^{22,24}$ Subjects, aged between 25 and 74 years and recruited between 1999 and 2001, were enrolled in an MR substudy and examined between June 2013 and September 2014 at the KORA study center. ${ }^{22}$ Subjects were excluded if they had any contraindications to either MR or gadolinium contrast administration. In addition, we included only subjects in this specific subanalysis with a complete set of all analyzed MR parameters. Thus, of overall 400 subjects who underwent whole-body MR imaging examinations, 157 participants were excluded due to incomplete MR imaging data sets (Supplementary Figure 1, Supplemental Digital Content 1, http://links.lww.com/RLI/A370).

While differences in single imaging markers of the overall cohort have been published previously, ${ }^{22}$ the current analysis is tailored to the assessment of comprehensive whole-body MR imaging phenotypic markers.

\section{Covariates}

Subjects of the KORA cohort were reexamined between June 2013 and September 2014 at the KORA study center. ${ }^{22}$ An oral glucose tolerance test was performed to all participants who had not been diagnosed for type 2 diabetes. According to the World Health Organization guideline $^{25}$ and as described previously, ${ }^{22,24}$ subjects were stratified into prediabetes, diabetes, and controls. Subjects who had been diagnosed with diabetes $\geq 7$ years ago were defined as long-term diabetes patients, based on the median duration of diabetes subjects in our study population $(\mathrm{n}=25)$, subjects with diabetes below this period were assigned to the group of short-term diabetes.

Other established risk factors such as hypertension, smoking, or increased body mass index (BMI) were collected in standardized fashion as part of the KORA study design and are described elsewhere. ${ }^{22,24}$

\section{Magnetic Resonance Imaging-Acquisition and Image Analysis}

As described previously, MR scans were performed with a $3 \mathrm{~T}$ whole-body MR system (Magnetom Skyra; Siemens AG, Healthcare Sector, Erlangen, Germany). ${ }^{22}$ Details on the MR protocol comprising sequences of the whole body including the brain, cardiovascular system, and adipose tissue compartments are provided in Supplementary Table 1, Supplemental Digital Content 2, http://links.lww.com/RLI/ A $371 .{ }^{22}$ All analyses were performed in blinded fashion by 2 independent readers each (overall 6 independent readers with $\geq 3$ years of experience) unaware of the diabetic group and clinical covariates on dedicated offline workstations. In case of discrepancy, a consensus reading was performed. To allow for improved interpretability, cut-points representing binary normal versus abnormal results of MR parameters per subject were derived individually and included either known pathologic thresholds (ie, LGE or hepatic PDFF), established grading systems (ie, ARWMC), or the highest 75 th percentile of a similar population (ie, VAT).

\section{Assessment of White Matter Lesions}

According to the ARWMC rating scale adapted from the Fazekas scale, FLAIR sequences were evaluated for T2 hyperintense area $\geq 5 \mathrm{~mm}$ lesions in 5 brain areas per hemisphere. ${ }^{18,26}$ A total ARWMC value ranging from 0 to 30 was derived. As severity of ARWMC and diabetes is associated with cognitive decline, all subjects with a severity of $\mathrm{ARWMC} \geq 1$ (mild/moderate) were categorized as abnormal. ${ }^{15}$

\section{Gadolinium Enhancement of the Myocardium}

Late gadolinium enhancement was acquired on fast low-angle shot inversion recovery sequences in short-axis stack and a 4-chamber view 10 minutes after administration of gadopentetate dimeglumine (0.2 mmol/kg, Gadovist; Bayer Healthcare, Berlin, Germany). For the assessment of the presence and distribution pattern of LGE, the 17-segment model of the American Heart Association was used. ${ }^{27}$ As LGE seems to be associated with adverse outcome in cardiomyopathy, the presence of LGE in any myocardial segment was considered as abnormal. ${ }^{21}$

\section{Assessment of Left Ventricular Function}

Cine-SSFP sequences were evaluated semi-automatically using commercially available software (cvi42, Circle Cardiovascular Imaging, Calgary, Canada) providing established LV volumetric data. The LVRI was calculated by the ratio of the LV mass to the LV end-diastolic volume. ${ }^{28}$ Left ventricular remodeling index $>1.3$ represents architectural and functional changes in myocardium and was considered as abnormal. ${ }^{20}$

\section{Assessment of Carotid Plaque}

Presence and measures of atherosclerotic plaque in the common carotid artery, at the carotid bulb, and in the proximal internal carotid artery on both sides were determined on black-blood T1-weighted fatsuppressed sequences. ${ }^{29}$ Any type of carotid plaque (type I, type III, type IV/V and type VI/VII) was considered as abnormal. ${ }^{29}$

\section{Assessment of Hepatic PDFF}

For the purpose of quantification of the hepatic PDFF, a multiecho VIBE T1-weighted sequence for determination of hepatic PDFF by accounting for confounding effects of $\mathrm{T} 2 *$ decay and the spectral complexity of fat were performed. ${ }^{30}$

Hepatic PDFF was classified according to the estimated hepatic PDFF thresholds for dichotomized hepatic steatosis scoring system for NAFLD from the nonalcoholic steatohepatitis clinical research network ancillary study: grade $0(<6.4 \%$ hepatic PDFF), grade $1(\geq 6.4$ to $<17.4 \%)$, grade $2(\geq 17.4$ to $<22.1 \%)$, and grade $3(\geq 22.1 \%)^{8}$; hepatic steatosis grade $\geq 1$ were considered as abnormal. ${ }^{6,31}$

\section{Assessment of Visceral Abdominal Adipose Tissue}

Visceral adipose tissue volume was measured from the femoral head to the cardiac apex, indicated in liter. High VAT levels seem to be related to adverse metabolic risk profiles, but studies about threshold levels associated with higher risk profiles are lacking. Thus, the determined cutoff value in our generated score is related to the $75 \%$ percentile volumebased VAT level of the healthy control group identified within the largescale UK Biobank Imaging Study (median, $1.32[0.86-1.79] \mathrm{L} / \mathrm{m}^{2}$ ). ${ }^{32}$ Visceral adipose tissue levels $\geq 1.79 \mathrm{~L} / \mathrm{m}^{2}$ were considered as abnormal. 


\section{Multiorgan MR Phenotypic Score}

An unweighted numeric summation score representing the extent of organ areas affected by subclinical disease as measured by MR was calculated by the sum of parameters exceeding the defined thresholds (range, 0-6). To characterize the distribution of the summation score, mean and standard deviation were calculated in addition to a categorization into low (score $\leq 1$ ), intermediate (score $=2$ ), and high ( score $\geq 3$ ).

\section{Statistical Analysis}

Subject demographics and cardiovascular risk factors are presented as arithmetic means and standard deviations for continuous variables and counts (percentages) for categorical variables. Differences in dichotomized MR features according to diabetes group were assessed by $\chi^{2}$ test. Differences in continuous MR features and in the multiorgan MR phenotypic score among diabetes groups were evaluated by one-way analysis of variance (ANOVA). Pairwise comparisons of multiorgan MR phenotypic score between short-/long-term diabetes and prediabetes were Bonferroni adjusted. Correlation between diabetes groups and multiorgan MR phenotypic score was tested by Spearman rank correlation coefficient.

To assess the association between diabetes groups and multiorgan MR phenotypic score, predicted score means with $95 \%$ confidence intervals (CIs) were calculated and compared by linear regression models adjusting for age, sex, smoking, BMI, hypertension, high-density lipoprotein, low-density lipoprotein, and triglycerides. Diabetes groups entered the model as a categorical variable with the 3 levels - control, prediabetes, and diabetes, with the control group as the reference group. Differences in baseline characteristics according to different multiorgan MR phenotypic score categories (low, intermediate, high as detailed above) were assessed by one-way ANOVA or $\chi^{2}$ test. The predictive power among the different MR parameters and the score was compared by fitting logistic regression models and comparing the c-statistics, which is equivalent to the area under the ROC curve ${ }^{33}$ using DeLongs nonparametric test of areas under the curve.

For the purpose of deriving underlying MR phenotypic patterns of the specific combinations of the dichotomized outcomes, unsupervised fuzzy clustering with a dissimilarity matrix given by Gower coefficient was used (Supplementary Figure 2, Supplemental Digital Content 1, http://links.lww.com/RLI/A370). ${ }^{34}$ Correlation between the resulting 2 clusters and glycemic status was assessed by $\chi^{2}$ test. Combinations of dichotomized outcomes associated to glycemic status were identified by LASSO regression. Variables that remained in the model with a nonzero coefficient after shrinkage were considered to be associated to glycemic status. ${ }^{35}$ The shrinkage parameter $\lambda$ was chosen as the minimum value after 10-fold cross validation. Fuzzy clustering and LASSO regression were carried out with $\mathrm{R}$ version 3.3.1 and packages cluster (v2.0.4) and glmnet (2.0-5). A 2-sided $P$ value of less than 0.05 was considered to indicate statistical significance.

\section{RESULTS}

Among 243 white subjects with complete MR data sets, 48 subjects were classified as prediabetes and 38 subjects had established diabetes mellitus ( $20 \%$ and $16 \%$, respectively). They were predominantly middleaged (55.6 \pm 8.9 years) with a slightly higher proportion of males $(62 \%)$ Further demographics and risk profiles are provided in Table 1.

Significantly higher ARWMC levels were found in subjects with diabetes and prediabetes compared with controls with normal glucose tolerance $(3.9 \pm 3.2$ vs $3.7 \pm 4.3$ vs $2.4 \pm 3.1, P=0.013$; respectively). Similar differences were found for LVRI, hepatic PDFF, and VAT (all $P<0.001)$. Carotid plaque was detected more often in subjects with

TABLE 1. Patient Demographic Characteristics and Cardiovascular Risk Factors

\begin{tabular}{|c|c|c|c|c|}
\hline & All & Control & Prediabetes & Diabetes \\
\hline Variable & $N=243$ & $\mathrm{n}=157$ & $n=48$ & $n=38$ \\
\hline Age, $y$ & $55.6 \pm 8.9$ & $54.2 \pm 8.8$ & $55.9 \pm 8.9$ & $61.3 \pm 7.5$ \\
\hline Male sex & $151(62.1 \%)$ & $89(56.7 \%)$ & $34(70.8 \%)$ & $28(73.7 \%)$ \\
\hline Height, cm & $172.5 \pm 9.2$ & $172.5 \pm 9.5$ & $173.2 \pm 9.7$ & $171.8 \pm 7.4$ \\
\hline Weight, kg & $82.2 \pm 14.7$ & $78.6 \pm 13.6$ & $90.0 \pm 11.6$ & $86.9 \pm 17.5$ \\
\hline BMI, $\mathrm{kg} / \mathrm{m}^{2}$ & $27.5 \pm 4.2$ & $26.3 \pm 3.6$ & $30.1 \pm 4.3$ & $29.3 \pm 4.7$ \\
\hline Waist-to-hip ratio & $0.9 \pm 0.1$ & $0.9 \pm 0.1$ & $1.0 \pm 0.1$ & $1.0 \pm 0.1$ \\
\hline Duration of diabetes, median [first quartile, third quartile], $\mathrm{y}$ & $7.0[5.0,12.0]$ & NA & NA & $7.0[5.0,12.0]$ \\
\hline $\mathrm{HbA} 1 \mathrm{c}, \%$ & $5.6 \pm 0.8$ & $5.3 \pm 0.3$ & $5.6 \pm 0.3$ & $6.7 \pm 1.5$ \\
\hline \multicolumn{5}{|l|}{ Smoking } \\
\hline Never-smoker & $85(35.0 \%)$ & $58(36.9 \%)$ & $16(33.3 \%)$ & $11(28.9 \%)$ \\
\hline Ex-smoker & $108(44.4 \%)$ & $63(40.1 \%)$ & $23(47.9 \%)$ & $22(57.9 \%)$ \\
\hline Smoker & $50(20.6 \%)$ & $36(22.9 \%)$ & $9(18.8 \%)$ & $5(13.2 \%)$ \\
\hline Systolic blood pressure, $\mathrm{mm} \mathrm{Hg}$ & $120.5 \pm 17.1$ & $116.9 \pm 15.6$ & $125.9 \pm 14.9$ & $128.2 \pm 21.3$ \\
\hline Diastolic blood pressure, $\mathrm{mm} \mathrm{Hg}$ & $75.4 \pm 9.8$ & $74.0 \pm 9.2$ & $78.9 \pm 9.6$ & $76.6 \pm 11.5$ \\
\hline Total cholesterol, mg/dL & $217.1 \pm 35.5$ & $215.9 \pm 34.7$ & $228.0 \pm 30.6$ & $208.2 \pm 41.9$ \\
\hline HDL cholesterol, mg/dL & $61.3 \pm 17.5$ & $64.3 \pm 17.2$ & $57.1 \pm 13.8$ & $54.1 \pm 20.0$ \\
\hline LDL cholesterol, mg/dL & $139.6 \pm 32.2$ & $138.9 \pm 30.4$ & $150.1 \pm 30.2$ & $129.2 \pm 38.3$ \\
\hline Triglycerides, mg/dL & $134.1 \pm 87.1$ & $111.3 \pm 71.3$ & $161.4 \pm 93.4$ & $193.8 \pm 101.3$ \\
\hline Hypertension & $77(31.7 \%)$ & $30(19.1 \%)$ & $21(43.8 \%)$ & $26(68.4 \%)$ \\
\hline Antihypertensive medication & $57(23.5 \%)$ & $23(14.6 \%)$ & $14(29.2 \%)$ & $20(52.6 \%)$ \\
\hline Antithrombotic medication & $12(4.9 \%)$ & $2(1.3 \%)$ & $3(6.2 \%)$ & $7(18.4 \%)$ \\
\hline Lipid lowering medication & $26(10.7 \%)$ & $8(5.1 \%)$ & $3(6.2 \%)$ & $15(39.5 \%)$ \\
\hline
\end{tabular}

Data are presented as mean \pm standard deviation for continuous variables and counts and percentages for categorical variables, unless otherwise indicated.

NA indicates not available; LDL, low-density lipoprotein; HDL, high-density lipoprotein. 
TABLE 2. Overview of Affected Organ Systems Among Subjects With Prediabetes, Diabetes, and Controls and Differences in Multiorgan MR Phenotypic Score Among Groups

\begin{tabular}{|c|c|c|c|c|c|}
\hline & All & Control & Prediabetes & Diabetes & \\
\hline & $N=243$ & $\mathbf{n}=\mathbf{1 5 7}$ & $\mathrm{n}=48$ & $\mathbf{n}=\mathbf{3 8}$ & $\boldsymbol{P}$ \\
\hline \multicolumn{6}{|l|}{ Brain } \\
\hline Total ARWMC Score, mean \pm SD & $2.9 \pm 3.4$ & $2.4 \pm 3.1$ & $3.7 \pm 4.3$ & $3.9 \pm 3.2$ & 0.013 \\
\hline Subjects with total ARWMC score $\geq 1, \mathrm{n}(\%)$ & $151(62.1 \%)$ & $88(56.1 \%)$ & $32(66.7 \%)$ & $31(81.6 \%)$ & 0.01 \\
\hline \multicolumn{6}{|l|}{ Cardiac } \\
\hline LVRI, mean \pm SD & $1.1 \pm 0.3$ & $1.0 \pm 0.2$ & $1.3 \pm 0.3$ & $1.4 \pm 0.4$ & $<0.001$ \\
\hline Subjects with LVRI >1.3, n (\%) & $51(21.0 \%)$ & $17(10.8 \%)$ & $18(37.5 \%)$ & $16(42.1 \%)$ & $<0.001$ \\
\hline Subjects with presence of LGE & $5(2.1 \%)$ & $2(1.3 \%)$ & $1(2.1 \%)$ & $2(5.3 \%)$ & 0.3 \\
\hline AHA myocardial segments involved & $1,2,4,4,4$ & 2,4 & 1 & 4,4 & \\
\hline \multicolumn{6}{|l|}{ Atherosclerosis } \\
\hline Subjects with presence of carotid plaque, n (\%) & $49(20.2 \%)$ & $26(16.6 \%)$ & $16(33.3 \%)$ & $7(18.4 \%)$ & 0.04 \\
\hline \multicolumn{6}{|l|}{ Visceral organ } \\
\hline $\mathrm{PDFF}$, mean $\pm \mathrm{SD}$ & $8.2 \pm 8.2$ & $4.9 \pm 4.8$ & $12.6 \pm 7.9$ & $16.2 \pm 11.4$ & $<0.001$ \\
\hline Subjects with $\mathrm{PDFF} \geq 6.4 \%, \mathrm{n}(\%)$ & $95(39.1 \%)$ & $33(21.0 \%)$ & $33(68.8 \%)$ & $29(76.3 \%)$ & $<0.001$ \\
\hline Subjects with grade 1 PDFF, n (\%) & $57(23.5 \%)$ & $25(15.9 \%)$ & $20(41.7 \%)$ & $12(31.6 \%)$ & \\
\hline Subjects with grade 2 PDFF, n (\%) & $15(6.2 \%)$ & $4(2.5 \%)$ & $4(8.3 \%)$ & $7(18.4 \%)$ & \\
\hline Subjects with grade 3 PDFF, n (\%) & $23(9.5 \%)$ & $4(2.5 \%)$ & $9(18.8 \%)$ & $10(26.3 \%)$ & \\
\hline \multicolumn{6}{|l|}{ Adipose tissue } \\
\hline $\mathrm{VAT}$, mean $\pm \mathrm{SD}, \mathrm{L} / \mathrm{m}^{2}$ & $4.4 \pm 2.6$ & $3.4 \pm 2.1$ & $5.8 \pm 2.2$ & $6.7 \pm 2.6$ & $<0.001$ \\
\hline Subjects with VAT $\geq 1.79 \mathrm{~L} / \mathrm{m}^{2}, \mathrm{n}(\%)$ & $83(34.2 \%)$ & $25(15.9 \%)$ & $29(60.4 \%)$ & $29(76.3 \%)$ & $<0.001$ \\
\hline Multiorgan MR phenotypic score, mean \pm SD & $1.79 \pm 1.35$ & $1.22 \pm 0.98$ & $2.69 \pm 1.50$ & $3.00 \pm 1.04$ & $<0.001$ \\
\hline
\end{tabular}

Data are presented as mean \pm standard deviation for continuous variables and counts and percentages for categorical variables, unless indicated otherwise. $P$ values are from $\chi^{2}$ test or one-way ANOVA. PDFF grade 1 : PDFF $\geq 6.4 \%$ but $<17.4 \%$. PDFF grade 2 : PDFF $\geq 17.4 \%$ but $<22.1 \%$. PDFF grade 3 : PDFF $\geq 22.1 \%$.

AHA indicates American Heart Association; MR, magnetic resonance; ARWMC, age-related white matter changes; LVRI, left ventricular remodeling index; LGE, late gadolinium enhancement; PDFF, proton density fat fraction of the liver; VAT, visceral adipose tissue.

prediabetes and diabetes compared with controls $(P=0.04)$. The presence of LGE was rare (2.1\% of all 243 subjects).

The prevalence of these MR features, dichotomized based on previously published cutoffs, are detailed in Table 2.

\section{Multiorgan MR Phenotypic Score}

On average, the multiorgan MR phenotypic score was $1.79 \pm 1.35$ and ranged from 0 to 5 . Subjects with prediabetes and diabetes had significantly higher scores compared with controls with normal glucose tolerance $(P<0.001$; Table 2, Supplementary Figure 3, Supplemental Digital Content 1, http://links.lww.com/RLI/A370). These differences remained significant after multivariable adjustment for age, sex, smoking, BMI, hypertension, high-density lipoprotein, low-density lipoprotein, and triglycerides $(P<0.001$, Fig. 1). The multiorgan MR phenotypic score provided the highest discriminatory power to predict prediabetes and diabetes as compared with single MR features (area under the curve, 0.824) and provided the highest risk estimate (odds ratio [OR], 25.92; 95\% CI, 10.83-62.05, for high vs low score, Table 3).

When comparing subjects with long-term, short-term, and newly diagnosed diabetes mellitus, there were no differences in MR phenotypic score between the groups (MR phenotypic score: $3.1 \pm 1.0 \mathrm{vs} 2.9 \pm 1.2 \mathrm{vs}$ $2.8 \pm 1.1, P=0.16$, for long-term vs short-term vs newly diagnosed diabetes mellitus; respectively).

\section{MR Phenotype Pattern between Subgroups}

The frequency distribution of MR features in controls and subjects with prediabetes and diabetes stratified by extent of multiorgan MR phenotypic score is shown in Figures 2 and 3. Among controls with low score, a high prevalence of elevated ARWMC was found (47,5\%), while only in a small proportion of these subjects, hepatic PDFF, carotid

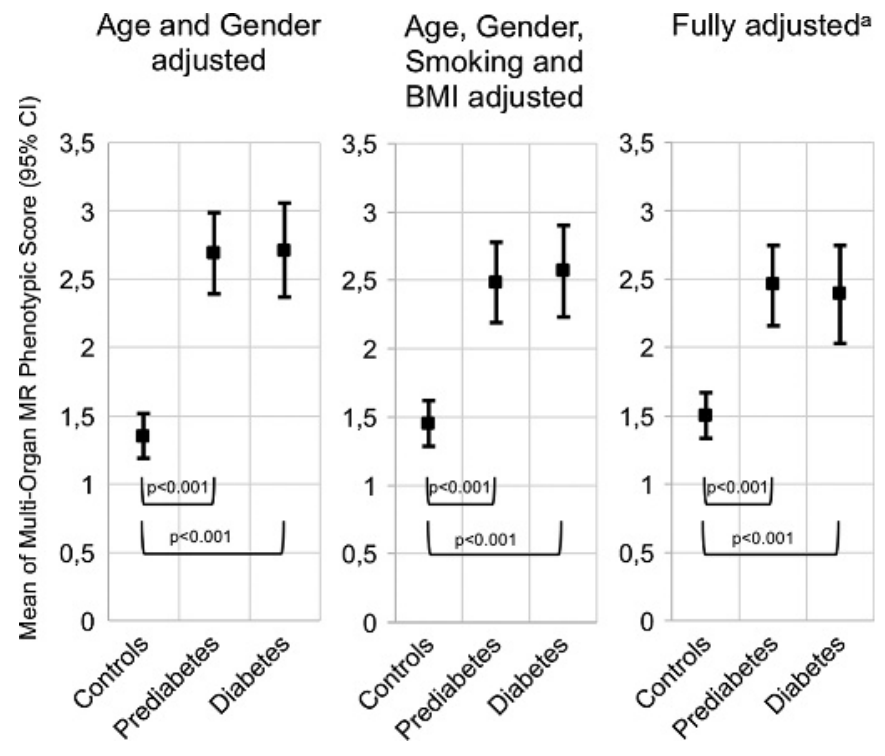

FIGURE 1. Predicted means of multiorgan MR phenotypic score after adjustment by linear regression analysis. Higher means of multiorgan MR phenotypic score were independently associated with impaired glucose metabolism $(P<0.001)$. *Adjusted for age, sex, smoking, body mass index, hypertension, high-density lipoprotein, low-density lipoprotein, and triglycerides. 
TABLE 3. Risk and Discriminatory Power of MR Phenotypic Score and Single MR Parameters to Predict Metabolic Disease State (Prediabetes and Diabetes) Adjusted for Age and Sex

\begin{tabular}{|c|c|c|c|c|c|}
\hline & OR & $95 \%$ CI & $P$ & $\mathbf{A U C}$ & $P^{*}$ \\
\hline \multicolumn{6}{|l|}{ MR Phenotypic Score } \\
\hline Numeric & 3.03 & $2.21-4.16$ & $<0.001$ & 0.824 & \\
\hline Intermediate vs low & 2.38 & $1.04-5.42$ & 0.0392 & 0.812 & 0.1 \\
\hline High vs low & 25.92 & $10.83-62.05$ & $<0.001$ & & \\
\hline \multicolumn{6}{|l|}{ Single MR parameters } \\
\hline ARWMC & 1.83 & $1-3.38$ & 0.0513 & 0.674 & $<0.00$ \\
\hline LVRI & 4.64 & $2.34-9.21$ & $<0.001$ & 0.728 & $<0.00$ \\
\hline Plaque & 1.59 & $0.82-3.09$ & 0.173 & 0.673 & $<0.00$ \\
\hline LGE & 2.12 & $0.34-13.31$ & 0.422 & 0.670 & $<0.00$ \\
\hline PDFF & 8.99 & $4.68-17.26$ & $<0.001$ & 0.787 & 0.0 \\
\hline VAT & 10.11 & $5.04-20.27$ & $<0.001$ & 0.774 & 0.0 \\
\hline
\end{tabular}

$* P$ value from DeLongs test if AUC of MR phenotypic Score (numeric) is larger than AUC of each single parameter.

OR indicates odds ratio; CI, confidence interval; AUC, area under the curve; ARWMC, age-related white matter changes; LVRI, left ventricular remodeling index; LGE, late gadolinium enhancement; PDFF, proton density fat fraction of the liver; VAT, visceral adipose tissue.

plaque, VAT, or myocardial changes were detected $(6.9 \%, 5.0 \%, 2.0 \%$, $4.0 \%$, and $0.0 \%$ for hepatic PDFF, carotid plaque, VAT, LVRI, and LGE, respectively). The prevalence increased continuously among controls resulting in highest prevalence of hepatic PDFF and VAT in control subjects with high multiorgan MR phenotypic score $(85.7 \%$ and $78.6 \%$, respectively). Similarly, among subjects with prediabetes and diabetes and low score, the prevalence of ARWMC and hepatic PDFF was highest (31.2\% and $18.8 \%$, respectively). Among subjects with prediabetes and diabetes and high score, the prevalence of hepatic PDFF and VAT was highest (91.1\% and $92.9 \%$, respectively). We found significantly higher ARWMC, LVRI, hepatic PDFF, and VAT levels in subjects with prediabetes and diabetes and high score compared with subjects with prediabetes and diabetes and low score (Fig. 3) (all $P<0.001$ ).

\section{MR-Based Cluster Analysis}

Assessing the combination of normal and abnormal MR parameters (ARWMC, hepatic PDFF, VAT, LVRI, carotid plaque, and LGE) in each subject, unsupervised fuzzy clustering revealed 2 different

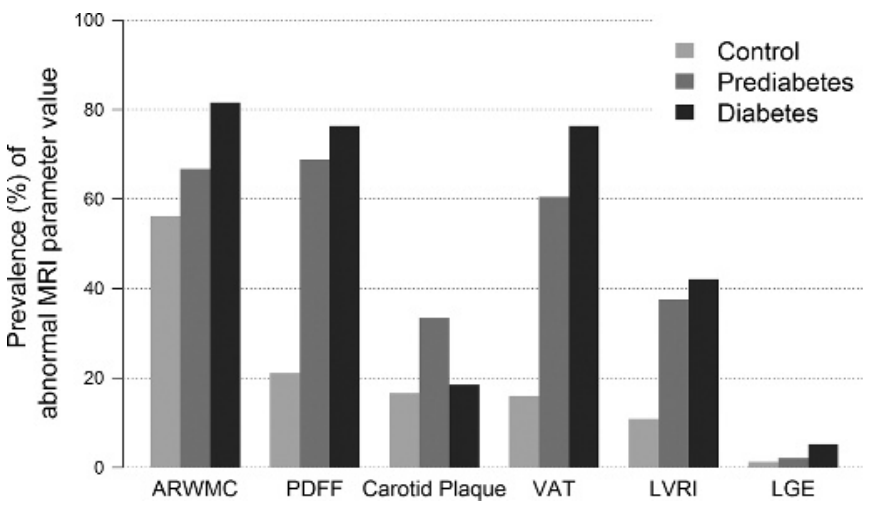

FIGURE 2. Prevalence of MR-based parameters among controls, prediabetes, and diabetes patients. ARWMC indicates age-related white matter changes; PDFF, hepatic proton density fat fraction; VAT, visceral adipose tissue; LVRI, left ventricular remodeling index; LGE, late gadolinium enhancement.

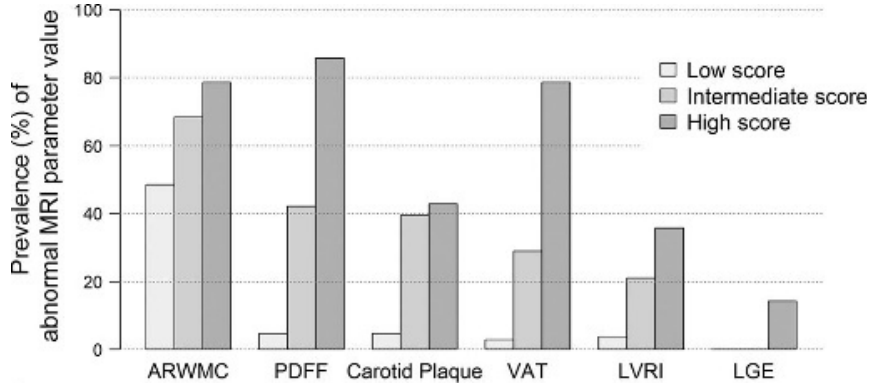

A

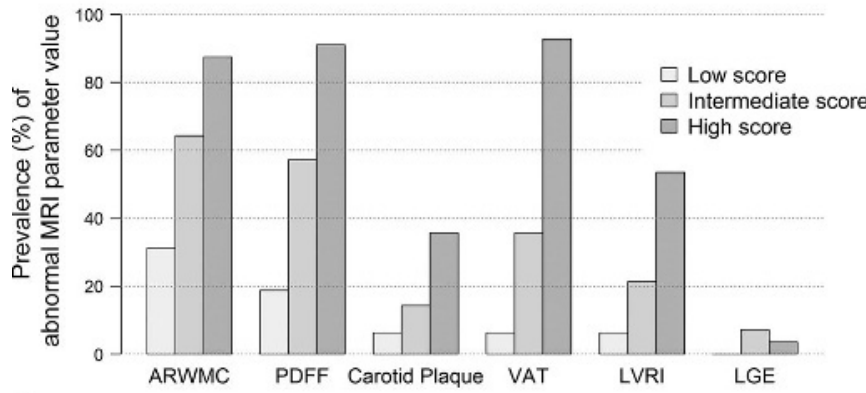

B

FIGURE 3. Frequency distribution of MR-based parameters among low-, intermediate-, and high-score ranges $(0-1,2$, and $\geq 3$, respectively) between controls (A) and patients with prediabetes and diabetes (B). ARWMC denotes age-related white matter changes; PDFF, hepatic proton density fat fraction; VAT, visceral adipose tissue volume; LVRI, left ventricular remodeling index; LGE, late gadolinium enhancement.

multiorgan phenotypic clusters (Supplementary Figure 2, Supplemental Digital Content 1, http://links.lww.com/RLI/A370) which were significantly associated with glycemic status $(P<0.001)$. After adjustment for high multiorgan MR phenotypic score, cluster II was still independently associated with prediabetes/diabetes group (OR, 10.05; 95\% CI, 3.75-27.00, $P=<0.001)$. Within the group with low multiorgan MR phenotypic score, cluster II was also significantly associated to diabetes status (OR, 2.49; 95\% CI, 1.00-6.17, $P=0.049)$. No further prediabetes-specific cluster could be identified. Figure 4 presents the distribution of the observed 33 combinations of dichotomized MR parameters (of theoretically possible 64 combinations) that occurred in the sample according to glycemic status and cluster membership. LASSO regression revealed that the MR feature combinations of only abnormal ARWMC and of only abnormal ARWMC plus carotid plaque were associated with normal controls, while all MR feature combination patterns associated with prediabetes/diabetes included hepatic PDFF and VAT beside other abnormal MR parameters (Figs. 4, 5).

\section{DISCUSSION}

In this sample from the general population, we characterized multiorgan involvement of subclinical disease phenotypes between subjects with impaired glucose metabolism and controls. While our results demonstrate that the overall prevalence and distribution of MR variables representing metabolic alterations is significantly elevated among subjects with prediabetes and diabetes as compared with controls, our findings also indicate that metabolic organ alterations, such as elevated LVRI, hepatic PDFF, and increased VAT volumes, are mainly affected in subjects with higher scores. We also identified distinctive multiorgan phenotypic patterns in subjects with prediabetes or diabetes status, which are specific for metabolic disease.

Metabolic organ alterations, for example, the fatty degeneration of internal organs such as the liver (ie, NAFLD), seem to be associated with chronic inflammatory state, which affects the adipose tissue and 


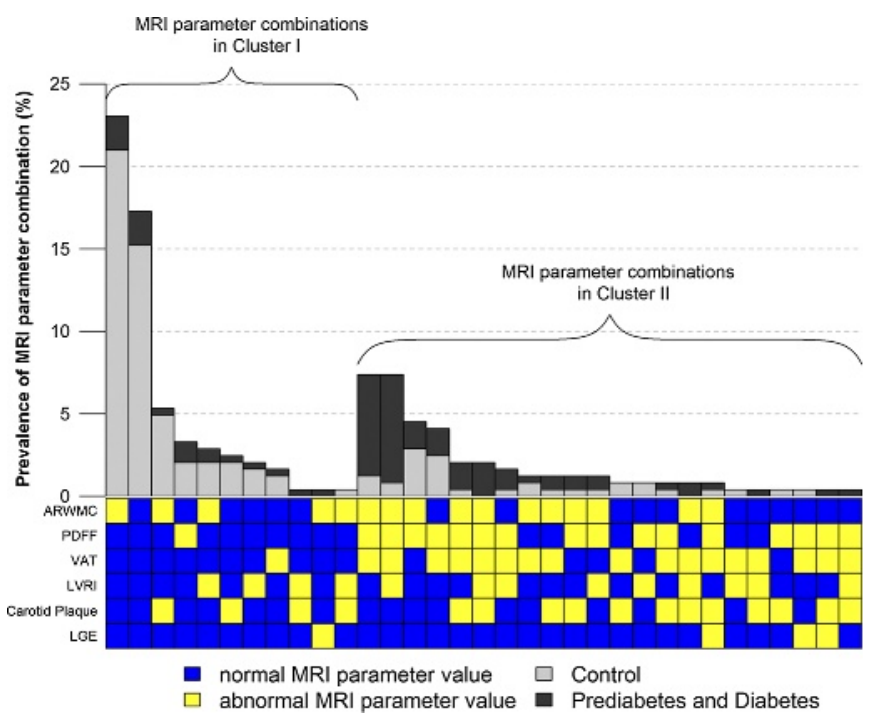

FIGURE 4. Distribution of specific combinations of dichotomized MR phenotypic parameters. Each MR phenotypic parameter is binary and may either be normal/negative (blue) or abnormal/positive (yellow). Among 64 possible combinations of MR phenotypic parameters, 33 combinations were observed. Each column in the figure represents the prevalence of a specific combination in the whole sample. The percentage of subjects with prediabetes/diabetes is shown as black; the gray columns display the control group. There were 2 clusters of MR phenotype patterns associated with glycemic status $(P<0.001)$ independent of the MR score. Cluster II was independently associated with diabetes state (odds ratio, 2.49; 95\% confidence interval, 1.00-6.17; $P=0.049$ ). ARWMC indicates age-related white matter changes; PDFF, hepatic proton density fat fraction; VAT, visceral adipose tissue volume; LVRI, left ventricular remodeling index; LGE, late gadolinium enhancement.

contribute to the complex pathomechanism of the development of an abnormal glucose metabolism, increased oxidative stress, dyslipidemia, and endothelial dysfunction with accelerated atherosclerosis and microangiopathy and macroangiopathy. ${ }^{6}$ These changes lead to liver diseases and dysfunctional cardiometabolic phenotypes, which result in a higher risk for adverse cardiovascular events and mortality. ${ }^{6,10}$ In addition, high levels of VAT are associated with cardiometabolic risk factors and are accompanied with the development of dyslipidemia and impaired glucose metabolism. ${ }^{8,9,36}$

In summarizing individual findings in multiple organs, we confirm earlier evidence pertaining to single findings. Specifically, hepatic PDFF is known to be associated with VAT and adverse metabolic risk profiles, independent of standard anthropometric indexes such as the BMI. $^{36}$ Similarly, white matter lesions, supposed as result of cerebrovascular origin, were found to be independent risk factors for cognitive decline and previous studies observed an association between changes in the white matter of the brain and diabetic disease. ${ }^{15,37}$ In addition, LGE, representing myocardial fibrosis, and LVRI, a measure for structural changes in affected myocardium, were found to be reliable factors for the assessment of structural and functional changes ${ }^{19,21}$ and represent strong predictors of adverse cardiovascular outcome and impaired cardiac function. ${ }^{19,21}$ While we found that these specific MR phenotypes are elevated in subjects with diabetes and prediabetes, we found more subjects with higher LVRI indices in the high score as compared with subjects in the low-score group. However, we found a high percentage of ARWMC among controls with low MR score, which can be traced back to the fact that white matter lesions can also be caused by inflammatory vessel processes and hypertensive diseases, which may lead to the assumption that ARWMC represents an early detectable
MR pattern with high prevalence in adult population, representing cardiovascular changes. ${ }^{38,39}$ Interestingly, we found a substantially lower prevalence of $5.3 \%$ of LGE in our diabetic population as compared with a prior analysis in Korean population (15\%), which may be attributable to the fact that these subjects underwent clinically indicated MR imaging due to suspected coronary artery disease and were retrospectively included. ${ }^{40}$ Also, in contrast to Yoon et al, we excluded subjects with history of stroke or peripheral artery disease, which may further decrease likelihood of presence of disease. As such, our results may be generalizable to a general asymptomatic population without prior known cardiovascular disease only.

Our findings may indicate that risk factors such as high adipose tissue levels or fatty liver disease may occur relatively early within the course of diabetes disease and may lead to the end point of irreversible organic architectural and functional changes especially in the myocardium, caused by impaired glucose metabolism. ${ }^{8-10}$ In addition, there was no difference in multiorgan MR phenotypes between subjects with long-term and short-term or newly diabetes mellitus, which may be attributable to the overall high effectiveness of currently available treatment options once diagnosed in this sample from a western European general population.

More importantly, by utilizing an MR imaging approach, we performed a multiparametric assessment of different clusters representing different combinations of phenotypic patterns that occurred in our study sample. This cluster analysis reveals a number of relevant findings. First, subjects with prediabetes and diabetes were assigned to the same cluster (Supplemental Figure 2, Supplemental Digital Content 1, http:// links.lww.com/RLI/A370). In contrast, unsupervised cluster analysis could not identify a distinct phenotypic cluster for subjects with prediabetes, confirming the close metabolic relationship between the 2 hyperglycemic disease entities observed when applying the multiorgan MR phenotypic Score. Second, we identified distinctive clusters and specific phenotype patterns of healthy controls and subjects with prediabetes and diabetes. Subjects with normal glucose tolerance (healthy controls) mainly show MR parameter combinations of abnormal changes in ARWMC and carotid plaque only, with LVRI in higher score levels to some extent, whereas additional changes including hepatic PDFF and VAT are more likely in subjects with prediabetes and diabetes. This observation illustrates the distinctive multiorgan pattern in subjects with impaired glucose metabolism and outlines the importance of metabolic alterations such as hepatic PDFF and VAT representing the strongest contributors for the assessment of metabolic differences as compared with healthy controls. Third, the MR phenotypic cluster was associated with diabetes state, independent of a high multiorgan MR phenotypic score, thus providing incremental information in characterizing hyperglycemic disease manifestation as demonstrated by its superior discriminatory power compared with single MR parameter assessment. Overall, these results suggest that a comprehensive, detailed clustering-based assessment of subclinical MR phenotypes may provide incremental value in characterization of early metabolic changes and identifying the individual extent and risk profile of involvement of different organ systems, even in subclinical stages.

Our study has several limitations. First, our study assesses the cross-sectional association of imaging findings and clinical disease types. While this may be highly relevant for gaining more insights into the disease process, clinical relevance and consequence for patient management will need to be determined in longitudinal cohorts. Unfortunately, at this point in time, we have no outcome data available to determine the prognostic value of the MR phenotypic score. However, this MR phenotypic score provides a baseline for all subsequent analysis along the longitudinal course of our study and allows to integrate the multiorgan imaging into a sum estimate to display the extent of subclinical disease in patients with impaired glucose metabolism. Notably, studies on the definition of "normal versus abnormal" threshold levels of MR-based parameters derived from healthy cohorts are scarce, and there is a lack of uniform cutoff 


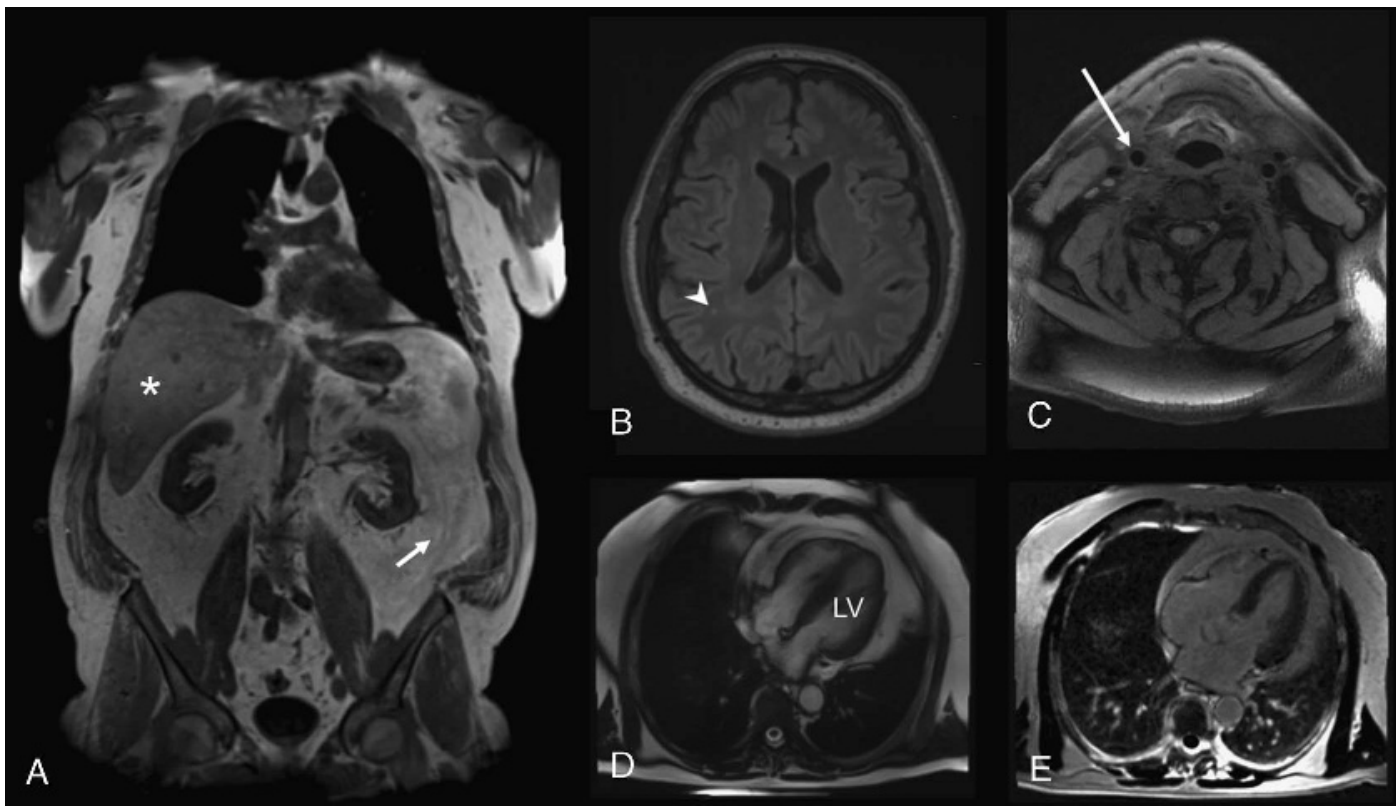

FIGURE 5. Imaging findings in a 61-year-old male as part of the study protocol. A, Two-point DIXON T1-weighted sequence for the assessment of visceral adipose tissue (VAT) volume from the femoral head to the cardiac apex (arrow) indicating high levels of VAT as well as hepatic proton density fat fraction (asterix, measured on multi-echo VIBE T1-weighted sequences). B, Fluid-attenuated inversion recovery sequences demonstrating mild white matter lesions (arrowhead). C, Atherosclerotic carotid plaque was determined on black-blood T1-weighted fat suppressed sequences in the common carotid artery (arrow), the carotid bulb, and the proximal internal carotid artery. D, Cine-SSFP sequences were evaluated for the calculation of volume and mass left ventricle (LV). F, late gadolinium enhancement was detected on fast-low-single-shot inversion recovery sequences 4-chamber view. The overall MR phenotypic score in this subject totaled 4.

levels associated with higher risk profiles. Thus, we applied heterogeneous definitions of thresholds, which were previously found to be strongly associated with impaired glucose metabolism and/or associated with higher cardiovascular risk and adverse outcome in an unweighted fashion. ${ }^{22}$ For instance, the presence of LGE was previously found to be associated with an 8-fold increased risk of an adverse cardiovascular event, whereas an ARWMC score $>1$ is associated only with mild or moderate risk for adverse cardiovascular outcomes. ${ }^{15,21}$ We applied $1.791 / \mathrm{m}^{2}$ as a threshold level for VAT, which represents the 75th percentile in a large-scale population-based healthy sample from UK Biobank Imaging Study and was associated with increased of risk for adverse outcome and corresponds to the 84 th percentile in our population. ${ }^{32}$ It is clear that the thresholds we applied as well as the unweighting of parameters are preliminary and will require further adjustment once novel pertaining research findings occur. Thus, further research is clearly warranted but our findings may serve as a hypothesis-generating reference. Finally, the study was conducted in a southern German general population, and all subjects were white, thus the generation of our results to different settings is limited.

In our study population without prior cardiovascular disease, subjects with prediabetes and diabetes have significantly higher multiorgan involvement of subclinical disease as compared with subjects with normal glucose tolerance and feature a diabetes-specific pattern of MR imaging phenotypes. These specific disease patterns are accentuated when performing cluster analysis, which revealed distinctive hyperglycemic multiorgan phenotypic clusters of subclinical disease manifestation in subjects with impaired glucose metabolism, containing more metabolic organ alterations as compared with control subjects including elevated levels of hepatic PDFF and VAT volume as well as structural cardiac changes such as LVRI. With this study, we demonstrate that diabetes and prediabetes is associated with a multiorgan footprint that can be identified and quantified by MR imaging. Furthermore, MR imaging may provide a more detailed assessment of the extent of subclinical disease and multiorgan alterations, which were previously shown to be associated with higher risk for cardiovascular events and adverse outcome and may therefore justify a more complex and costly imaging procedure. As such, MR imaging may provide detailed insights into metabolic disease process and pathogenesis and thus may enable an individual characterization of disease states and improve risk stratification.

\section{REFERENCES}

1. NCD Risk Factor Collaboration (NCD-RisC). Worldwide trends in diabetes since 1980: a pooled analysis of 751 population-based studies with 4.4 million participants. Lancet. 2016;387:1513-1530.

2. King H, Aubert RE, Herman WH. Global burden of diabetes, 1995-2025: prevalence, numerical estimates, and projections. Diabetes Care. 1998;21:1414-1431.

3. Danaei G, Lawes CM, Vander Hoorn S, et al. Global and regional mortality from ischaemic heart disease and stroke attributable to higher-than-optimum blood glucose concentration: comparative risk assessment. Lancet. 2006;368:1651-1659.

4. Aneja A, Tang WH, Bansilal S, et al. Diabetic cardiomyopathy: insights into pathogenesis, diagnostic challenges, and therapeutic options. Am J Med. 2008;121 748-757.

5. Shah RV, Abbasi SA, Heydari B, et al. Insulin resistance, subclinical left ventricular remodeling, and the obesity paradox: MESA (Multi-Ethnic Study of Atherosclerosis). J Am Coll Cardiol. 2013;61:1698-1706.

6. Bhatia LS, Curzen NP, Calder PC, et al. Non-alcoholic fatty liver disease: a new and important cardiovascular risk factor? Eur Heart J. 2012;33:1190-1200.

7. Stefan N, Kantartzis K, Haring HU. Causes and metabolic consequences of fatty liver. Endocr Rev. 2008;29:939-960.

8. Tang L, Zhang F, Tong N. The association of visceral adipose tissue and subcutaneous adipose tissue with metabolic risk factors in a large population of Chinese adults. Clin Endocrinol (Oxf). 2016;85:46-53.

9. Goodpaster BH, Krishnaswami S, Resnick H, et al. Association between regional adipose tissue distribution and both type 2 diabetes and impaired glucose tolerance in elderly men and women. Diabetes Care. 2003;26:372.

10. Cusi K. Nonalcoholic fatty liver disease in type 2 diabetes mellitus. Curr Opin Endocrinol Diabetes Obes. 2009;16:141-149.

11. Stefan N, Fritsche A, Schick F, et al. Phenotypes of prediabetes and stratification of cardiometabolic risk. Lancet Diabetes Endocrinol. 2016;4:789-798.

12. Kantartzis K, Machann J, Schick F, et al. The impact of liver fat vs visceral fat in determining categories of prediabetes. Diabetologia. 2010;53:882-889. 
13. Hsu JL, Chen YL, Leu JG, et al. Microstructural white matter abnormalities in type 2 diabetes mellitus: a diffusion tensor imaging study. Neuroimage. 2012; 59:1098-1105.

14. van Harten B, de Leeuw FE, Weinstein $\mathrm{HC}$, et al. Brain imaging in patients with diabetes: a systematic review. Diabetes Care. 2006;29:2539-2548.

15. Verdelho A, Madureira S, Moleiro C, et al. White matter changes and diabetes predict cognitive decline in the elderly: the LADIS study. Neurology. 2010;75: $160-167$.

16. Idilman IS, Keskin O, Celik A, et al. A comparison of liver fat content as determined by magnetic resonance imaging-proton density fat fraction and MRS versus liver histology in non-alcoholic fatty liver disease. Acta Radiol. 2016;57: 271-278.

17. Wintersperger BJ, Bamberg F, De Cecco CN. Cardiovascular imaging: the past and the future, perspectives in computed tomography and magnetic resonance imaging. Invest Radiol. 2015;50:557-570.

18. Fazekas F, Chawluk JB, Alavi A, et al. MR signal abnormalities at $1.5 \mathrm{~T}$ in Alzheimer's dementia and normal aging. AJR Am J Roentgenol. 1987;149: 351-356.

19. Yoon JH, Son JW, Chung H, et al. Relationship between myocardial extracellular space expansion estimated with post-contrast T1 mapping MRI and left ventricular remodeling and neurohormonal activation in patients with dilated cardiomyopathy. Korean J Radiol. 2015;16:1153-1162.

20. Gaasch WH, Zile MR. Left ventricular structural remodeling in health and disease: with special emphasis on volume, mass, and geometry. J Am Coll Cardiol. 2011;58:1733-1740.

21. Wu KC, Weiss RG, Thiemann DR, et al. Late gadolinium enhancement by cardiovascular magnetic resonance heralds an adverse prognosis in nonischemic cardiomyopathy. J Am Coll Cardiol. 2008;51:2414-2421.

22. Bamberg F, Hetterich H, Rospleszcz S, et al. subclinical disease burden as assessed by whole-body MRI in subjects with prediabetes, subjects with diabetes, and normal control subjects from the general population: the KORA-MRI study. Diabetes. 2017;66:158-169.

23. Bamberg F, Kauczor HU, Weckbach S, et al. Whole-body MR imaging in the german national cohort: rationale, design, and technical background. Radiology. 2015;277:206-220.

24. Holle R, Happich M, Löwel H, et al. KORA — a research platform for population based health research. Gesundheitswesen. 2005;67(suppl 1):S19-S25.

25. Press W. World Health Organization. Definition and diagnosis of diabetes mellitus and intermediate hyperglycemia. In: Publications of the World Health Organization. Geneva, Switzerland: World Health Organization; 2006.

26. Wahlund L, Barkhof F, Fazekas F, et al. A new rating scale for age-related white matter changes applicable to MRI and CT. Stroke. 2001;32:1318-1322.
27. American College of Cardiology Foundation Task Force on Expert Consensus Documents, Hundley WG, Bluemke DA, Finn JP, et al. ACCF/ACR/AHA NASCI/SCMR 2010 expert consensus document on cardiovascular magnetic resonance: a report of the American College of Cardiology Foundation Task Force on Expert Consensus Documents. J Am Coll Cardiol. 2010;55:2614-2662.

28. Lamb HJ, Beyerbacht HP, de Roos A, et al. Left ventricular remodeling early after aortic valve replacement: differential effects on diastolic function in aortic valve stenosis and aortic regurgitation. J Am Coll Cardiol. 2002;40:2182-2188.

29. Cai JM, Hatsukami TS, Ferguson MS, et al. Classification of human carotid atherosclerotic lesions with in vivo multicontrast magnetic resonance imaging. Circulation. 2002;106:1368-1373.

30. Hetterich H, Bayerl C, Peters A, et al. Feasibility of a three-step magnetic resonance imaging approach for the assessment of hepatic steatosis in an asymptomatic study population. Eur Radiol. 2015.

31. Kleiner DE, Brunt EM, Van Natta M, et al. Design and validation of a histological scoring system for nonalcoholic fatty liver disease. Hepatology. 2005;41: 1313-1321.

32. Dahlqvist Leinhard $\mathrm{O}$, Linge J, West J, et al Body composition profiling using MRI - normative data for subjects with diabetes extracted from the UK Biobank Imaging Cohort. Radiological Society of North America 2016 Scientific Assembly and Annual Meeting. Chicago, IL: 2016.

33. Hanley JA, McNeil BJ. The meaning and use of the area under a receiver operating characteristic (ROC) curve. Radiology. 1982;143:29-36.

34. Gower JC. A general coefficient of similarity and some of its properties. Biometrics. 1971;27:857-871.

35. Friedman J, Hastie T, Tibshirani R. Regularization paths for generalized linear models via coordinate descent. J Stat Softw. 2010;33:1.

36. Fox CS, Massaro JM, Hoffmann U, et al. Abdominal visceral and subcutaneous adipose tissue compartments: association with metabolic risk factors in the Framingham Heart Study. Circulation. 2007;116:39-48.

37. Weckbach S, Findeisen HM, Schoenberg SO, et al. Systemic cardiovascular complications in patients with long-standing diabetes mellitus: comprehensive assessment with whole-body magnetic resonance imaging/magnetic resonance angiography. Invest Radiol. 2009;44:242-250.

38. de Leeuw FE, de Groot JC, Oudkerk M, et al. Hypertension and cerebral white matter lesions in a prospective cohort study. Brain. 2002;125(pt 4):765-772.

39. van Dijk EJ, Prins ND, Vermeer SE, et al. C-reactive protein and cerebral smallvessel disease: the Rotterdam Scan Study. Circulation. 2005;112:900-905.

40. Yoon YE, Kitagawa K, Kato S, et al. Prognostic value of unrecognised myocardial infarction detected by late gadolinium-enhanced MRI in diabetic patients with normal global and regional left ventricular systolic function. Eur Radiol. 2013; 23:2101-2108 